\title{
Knowledge, attitudes, and practices concerning cockroach exposure among caregivers of children with asthma
}

\author{
Derek Werthmann*, Felicia A. Rabito and Charlie Reed
}

\begin{abstract}
Background: Cockroach allergen is one of the most important asthma triggers for children. There is an extensive body of research on interventions to reduce exposure. However, adherence to these interventions is low. Insight into the knowledge, attitudes, and practices (KAP) associated with cockroach remediation is needed. We assessed KAP using the Health Beliefs Model (HBM) as a framework for predicting behavior. This study aimed to assess the socio-demographic and psycho-social characteristics associated with cockroach KAP and to quantify the relationship between KAP and number of cockroaches in the home.

Methods: To identify factors associated with cockroach KAP a cross-sectional study was conducted using a structured questionnaire administered to caregivers of children with asthma in New Orleans, Louisiana. Positive scores in each metric of the KAP signified better cockroach knowledge, increased concern about cockroaches' impact on health (attitudes), and participation in recommended cockroach remediation practices (practices). To evaluate cockroach KAP scores as a predictor of cockroach exposure in the home, a cohort study was conducted among a sub-sample of participants.
\end{abstract}

Results: Fifty-six caregivers participated in the study. Participants had positive scores on all subscales of KAP with knowledge having a lower score compared to attitudes and practices. Cockroach knowledge was inversely correlated with age at asthma diagnosis $(\rho=-0.36, p=0.01)$. Caregivers identifying as black/African American had higher cockroach knowledge scores compared to other races (Median: $6.0 \vee 3.0 ; p=0.05$ ). Caregivers other than mothers had higher cockroach attitude scores (Median $6.5 \vee 4.0 ; p=0.003$ ) and total KAP scores (Median $18.0 \vee 14.0$; $p=0.05)$. Twenty-six participants completed the cohort study. Cockroach exposure was not significantly associated with higher cockroach knowledge, cockroach practice, or total KAP score. For attitude scores, participants in the highest quartile had significantly lower exposure ( $\beta$ : $-1.96,95 \% \mathrm{Cl}$ - $3.50-0.42)$ compared to those in the lowest quartile $(p=0.01)$.

Conclusions: Different socio-demographic and psycho-social factors were associated with the components of cockroach KAP. Greater concern about cockroaches (attitude) was significantly associated with reduced cockroach exposure. This highlights the importance of identifying the key elements of caregiver KAP to improve cockroach remediation among caregivers of children with asthma.

Keywords: Cockroach, Knowledge, Attitudes, Practices, Asthma, Health beliefs model

\footnotetext{
* Correspondence: dwerthma@tulane.edu

Department of Epidemiology, Tulane University School of Public Health and

Tropical Medicine, New Orleans, LA, USA
} 


\section{Background}

In the United States (U.S.) asthma is the most common childhood disease with an estimated prevalence of 6 million [1]. Asthma is also the leading cause of childhood morbidity [2] and a major contributor to school absenteeism with over 5.2 million lost school days annually due to asthma [3]. Families of low-income status are disproportionately affected. Compared to a prevalence of $8.3 \%$ in the general U.S. population, for those living below the federal poverty threshold the prevalence is $11.8 \%$ [4]. Clinical guidelines outline best practices for preventing asthma exacerbations and an important component is cockroach allergen avoidance. However, adherence to cockroach avoidance strategies is low (12-40\%) even when resources are supplied [5]. The reasons for low adherence are unclear.

Exposure to high levels of allergens in the home is related to poor asthma outcomes. Of the indoor allergens known to be related to asthma, exposure to cockroach, which is ubiquitous in urban environments, is strongly associated with severe asthma outcomes in children of low-income status [6-8]. Cockroach exposure is associated with significantly higher rates of hospitalization [9] and children sensitized and highly exposed to cockroach have almost double the number of unscheduled medical visits, and significantly more days of wheezing $(4.04 \mathrm{v}$ 3.21) and missed school (7.65 v 6.12) [10]. Cockroach allergen has been detected in $85 \%$ of inner-city homes in the U.S. and an estimated $60-80 \%$ of inner-city children with asthma are sensitized to cockroach [11]. Asthma management is complex requiring a multifaceted approach of both medication and environmental interventions. The most important environmental intervention is controlling exposure to allergens in the home [12]. It is an effective asthma management strategy; shown to be as effective at improving asthma outcomes as asthma medication [13]. However, data from the National Health Interview Survey show that less than half (46\%) of caregivers of children with asthma receive advice on environmental control [14]. Research also shows caregivers are not willing to adhere to the recommended interventions [15]. While there is a robust body of research examining factors related to medication adherence [16], research on the factors related to adherence to recommended environmental interventions, including cockroach remediation, is lacking. Given the effectiveness of cockroach remediation on improved asthma morbidity [17], this is an important knowledge gap.

Knowledge attitudes and practices (KAP) surveys are conducted to obtain health behavior information about what is known (knowledge), what is thought (attitudes), and what is done (practices) in specific populations. KAP surveys can identify knowledge gaps, cultural beliefs, behavioral patterns, and barriers. This information can be used to deepen the understanding of behaviors and plan the implementation of interventions [18]. Despite abundant data on the importance of cockroach allergen avoidance for children with asthma, the KAP regarding cockroach avoidance strategies and the impact cockroach KAP has on actual exposure in the home is unknown.

The Health Belief Model (HBM) was developed in the 1950 s by the U.S. Public Health Services to determine why people do not adopt preventative health measures [19]. It theorizes that health behavior is determined by personal beliefs, perceptions about the disease, and the availability of strategies to prevent its occurrence. The HBM's effectiveness in predicting behavior is welldocumented [20, 21]. The core of the HBM is an individual's perception of disease which is influenced by sociodemographic and psychosocial components. The HBM has six core constructs: perceived severity, perceived susceptibility, cues to action, perceived benefits, perceived barriers, and self-efficacy. Perceived severity of the disease and perceived susceptibility both contribute to an individual's perception of threat. Threat perception, along with perceived benefits, perceived barriers and self-efficacy determine the likelihood of a behavior being carried out [22]. Cues to action are independent triggers, either internal or external, that prompt an individual to action. We used the HBM as a framework to assess caregiver's qualities (sociodemographic and psychosocial) associated with cockroach KAP in order to explore the reasons for low adherence to cockroach avoidance strategies. We then assessed the impact of KAP regarding cockroaches on the level of cockroach exposure in the home.

The objective of this study was to describe the sociodemographic and psychosocial factors associated with cockroach KAP. A secondary objective was to assess the association between caregiver's cockroach KAP and actual exposure to cockroach in their home. The study hypothesis is that greater knowledge about cockroach (knowledge), higher concern about cockroach exposure (attitudes), and actions that promote cockroach remediation (practices) will result in reduced exposure to cockroaches in the home.

\section{Methods}

Using the HBM framework, (Fig. 1) we assessed the socio-demographic and psychosocial factors associated with cockroach KAP. Key socio-demographic factors include: race/ethnicity, education, marital status and housing factors (home ownership status, type of dwelling). Key psychosocial factors include stress, asthma knowledge, self-efficacy, social support, and medication beliefs. 


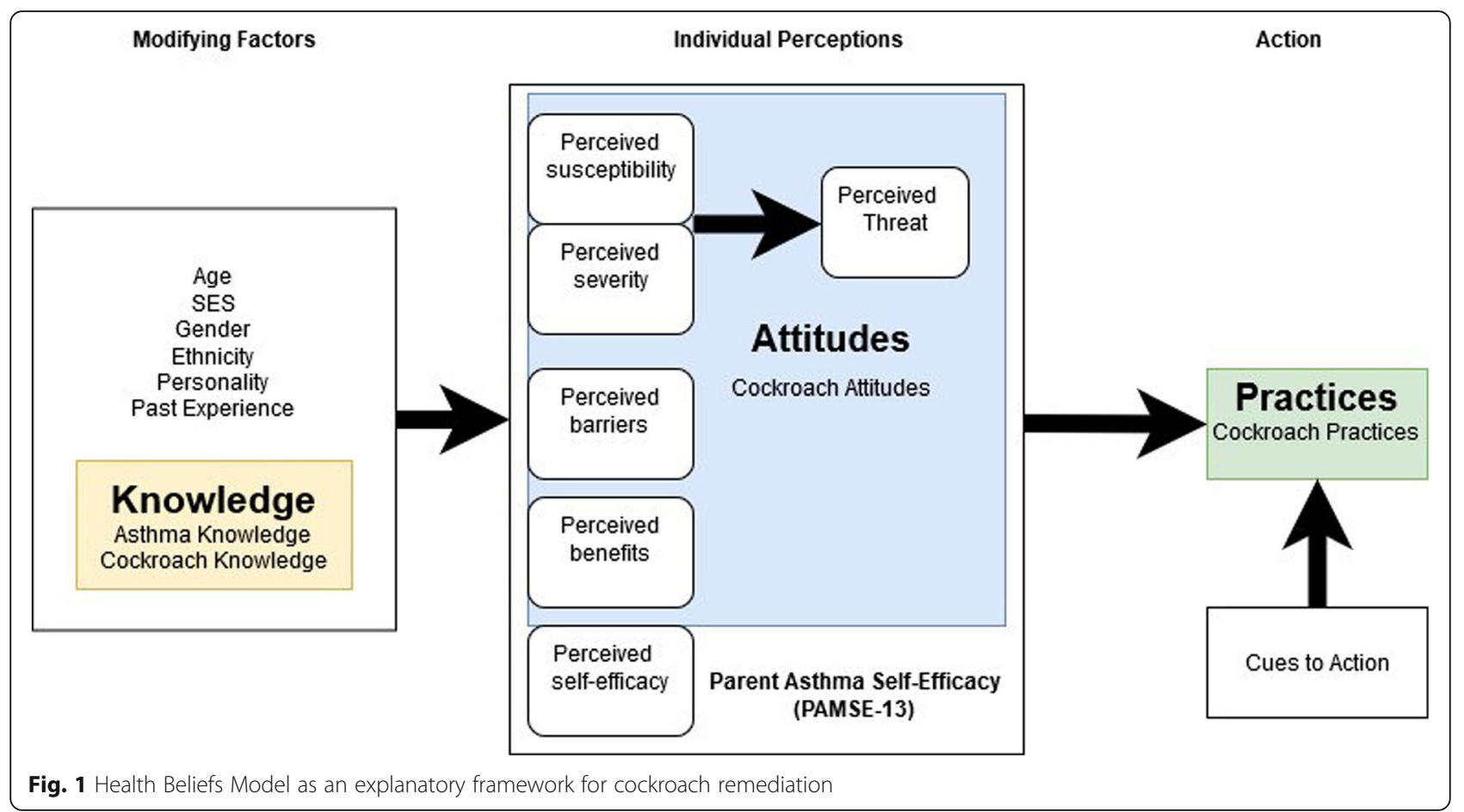

\section{Study design}

A cross-sectional study was performed to examine KAP of caregivers of children with asthma regarding the role of cockroach remediation on their child's asthma and to examine the correlation between cockroach KAP and specific socio-demographic and psycho-social factors. A cohort study was conducted among a sub-sample of participants to determine whether cockroach KAP scores are associated with cockroach exposure in the home. The cohort study consisted of administering the KAP survey followed by a home visit to measure cockroach counts. The study received approval from the Tulane University Biomedical Institutional Review Board and informed consent was obtained prior to data collection.

\section{Study participants}

Participants were recruited from community health fairs and the Children's Hospital of New Orleans Pediatric Pulmonary Clinic between August 2018 and April 2019. Eligibility requirements included being the caregiver of a child ( $<18$ years) with doctor-diagnosed asthma.

\section{Data collection}

\section{Knowledge, attitude and practices}

Knowledge, Attitude and Practices concerning cockroaches and their effect on asthma was assessed via an interviewer administered questionnaire adapted from a previous cockroach intervention study conducted in Toronto, Canada [23]. Study personnel trained in survey data collection administered the questionnaire. The KAP survey consists of three subscales: knowledge, attitudes and practices (Table 1). Knowledge parameters included health risks associated with exposure to cockroaches, factors contributing to cockroach infestation, and health risks associated with use of insecticides. The knowledge subscale consists of 20 statements with responses of "agree, disagree, and not sure". Correct responses were scored as 1 point and incorrect responses or a response of "not sure" were scored as negative 1, resulting in a scale ranging from -20 to 20 . Positive scores represent increasing knowledge while negative scores signify increasing inaccurate knowledge. The attitudes subscale of the KAP survey covered perceived effectiveness of cockroach control, health concerns about spraying insecticides to control cockroaches, and overall concern about cockroach exposure in the home. The attitudes scale consists of 10 statements with 4-item responses of "strongly agree, agree, disagree, or strongly disagree". Each item was scored as either $-1,-0.5,0.5$, or 1.0. The resulting scale ranges from -10 to 10 with increasing values being associated with greater acceptance of cockroach mitigation/remediation and greater concern about health risks associated with cockroaches. Finally, the KAP survey assessed caregivers' practices regarding the management and prevention of cockroach infestation. The practices scale consists of 10 items with 3-item responses of "often, sometimes, or rarely", these items were scored as $-1,0$, or 1 . The resulting scale ranges from -10 to 10 with positive values signifying practices for avoiding cockroaches and negative values indicating 
Table 1 Cockroach KAP questions and relationship to Health Beliefs Model

\section{Knowledge}

\section{Health risks of pesticides}

a. Exposure to some pesticides may interfere with the nervous system.

b. Smelling an odor does not necessarily mean over-exposure to pesticides.

c. Long-term exposure to pesticides, at low levels, is not safe.

d. Boric acid can be hazardous.

\section{The following are health risks and habits of cockroaches:}

a. Contact with cockroaches does not hurt skin.

b. Cockroaches do not give off harmful vapors.

c. Cockroaches do not worsen Tuberculosis conditions.

d. About $10 \%$ of people are allergic to cockroaches.

e. Persons with asthma are much more likely to have allergic reactions to cockroaches than non-asthmatics.

f. Cockroaches prefer dark, tight spaces.

\section{These factors contribute to cockroach infestation:}

a. Leaving grease on the stove overnight.

b. Leaving dirty dishes out overnight.

c. Keeping windows open.

d. Leaving food debris on the floor overnight.

e. Storing food in open packages in the cupboard.

f. Having a leaky tap.

g. Having a toilet that runs all the time.

h. Storing open bags of pet food.

i. Not having cracks or holes sealed around plumbing.

\section{Familiarity with cockroach bait method}

a. Cockroach bait is one type of pest control method

\section{Effectiveness/desirability of alternatives}

a. Cockroach baiting can be just as effective as spraying in controlling roaches.

b. Cockroach baiting can be just as convenient as spraying in controlling roaches.

c. I support safer methods other than spraying, even if they are slower-acting.

d. I expect the safest method of pest control to be used, even if it is twice as expensive.

\section{Concern about spraying}

a. I have concerns about spraying in the home.

b. Spraying is not the best way to eliminate cockroaches.

\section{Concern about Cockroaches}

a. I have concerns about health risks from cockroach infestation.

b. I have concerns about seeing a cockroach in my home.

c. I have concerns about cockroaches in my home, even if not visible.

d. Cockroaches may be a problem, even in homes where people are tidy.

\section{Recognition/management of cockroach problems}

a. I would not just put up with cockroach problems; I would do something.

b. I would not use my own pest control product such as a spray or bait.

\section{Prevention of cockroach infestation}
a. I clean crumbs soon after eating.
b. I clean dishes soon after eating.
c. I sweep the kitchen floor before bedtime.

Health Beliefs Model Construct

Knowledge

Knowledge

Knowledge

Knowledge

Knowledge

Knowledge

Knowledge

Knowledge

Knowledge

Knowledge

Knowledge

Knowledge

Knowledge

Knowledge

Knowledge

Knowledge

Knowledge

Knowledge

Knowledge

Knowledge

Attitudes: Perceived Barriers

Attitudes: Perceived Barriers

Attitudes: Perceived Barriers

Attitudes: Perceived Barriers

Attitudes: Perceived Benefits

Attitudes: Perceived Benefits

Attitudes: Perceived Susceptibility

Attitudes: Perceived Severity

Attitudes: Perceived Severity

Attitudes: Perceived Susceptibility

Practice

Practice

Practice

Practice

Practice 
Table 1 Cockroach KAP questions and relationship to Health Beliefs Model (Continued)

\begin{tabular}{ll}
\hline Knowledge & Health Beliefs Model Construct \\
\hline d. I store food containers in the cupboard. & Practice \\
e. I clean grease off the stove before bedtime. & Practice \\
f. I rinse food off dishes if left unwashed overnight. & Practice \\
g. I thoroughly clean the kitchen/bathroom weekly. & Practice \\
h. I watch for cockroaches which might be brought home with groceries. & Practice \\
\hline
\end{tabular}

more practices that promoted cockroach infestation. The overall KAP score is the sum of the 3 sub-scales, ranging from -40 to 40 .

\section{Cockroach exposure}

Cockroach exposure was measured in a subsample of participants to assess the association between KAP score and the presence of cockroaches in the home. Cockroach exposure was defined as the number of cockroaches trapped (counts) in the home over a three-day period. Cockroach count data were obtained during a home visit that occurred within seven days of the participant's completion of the KAP survey. Cockroach counts were obtained by study staff following protocols developed by researchers at North Carolina State University [24]. Briefly, an initial inspection of the home was performed, and study staff drew a layout map of the kitchen, living room, and the bedroom of the child with asthma. Next, a visual assessment of the home was conducted for any signs of cockroaches. The location and approximate number were recorded. Study personnel placed six pheromone sticky traps (Victor Roach Pheromone Traps, Woodstream, Lititz PA) in each room. The position of the traps was documented on the room diagram. Study personnel returned three days later to retrieve the traps. Traps were brought to the study laboratory where cockroaches were speciated and enumerated by trained study personnel. Data were recorded on a trap collection form.

\section{Socio-demographic data}

To assess the factors influencing cockroach KAP, sociodemographic and household data were collected via survey questionnaire using a structured instrument administered by study personnel. Data collected included: race/ethnicity of the caregiver, caregiver education level and marital status, type of residence, and age of the child at asthma diagnosis. The questionnaires were administered in person at the time of recruitment.

\section{Psycho-social measures}

Psychosocial factors associated with childhood asthma management were assessed using validated survey instruments [25-28] administered by study personnel either at the time of enrollment or at the home visit. To assess caregivers' beliefs about asthma medication, an important component of asthma management, the Beliefs about Medicine Questionnaire (BMQ) was administered. The BMQ assesses parents' beliefs and worries about children taking medication for their disease. It is an 18-item questionnaire with a four-factor structure (Specific-Necessity, Specific-Concerns, General-Harm, and General-Overuse). All items have a 5 point Likert answer option. Higher scores indicate stronger beliefs about the corresponding subscale (i.e. positive beliefs concerning medication use and negative beliefs about medication for the concerns, harm, and overuse scales). Social support is an important factor when carrying out health related behaviors. Therefore, the Medical Outcomes Study Social Support Survey Scale (MOS-SSS) was administered to each caregiver. The MOS-SSS assesses the extent to which a person has the support of others to face stressful situations. It consists of 6 items answered by means of a five-point Likert scale with scores ranging from 6 to 30. Higher scores indicating greater social support. Self-efficacy is one of the six key constructs of the HBM. To assess this construct the Parent Asthma Management Self-efficacy survey (PAMSE13) was administered. The PAMSE-13 is a 13-item scale, consisting of two sub-scales, asthma management and asthma prevention, with 5-point Likert responses ranging from 13 to 65 . Higher scores indicating greater selfefficacy for each sub-scale. Caregiver stress has a negative impact on asthma outcomes and the ability to perform asthma management behaviors [29]. Caregiver stress was measured via Cohen's Perceived Stress Scale (PSS) short-form, a 4-item scale scored with 4-point Likert scale. Scores range from 0 to 16 with higher scores indicating greater perceived stress.

\section{Asthma knowledge}

Knowledge is a key construct of the HBM, and a caregiver's knowledge of asthma is an important consideration when assessing cockroach KAP. Asthma knowledge was assessed using a questionnaire developed by Martinez et al. [30] The tool has been found to be reliable for quantifying the baseline level of asthma knowledge of parents of children with asthma. It consists of 17 questions and is scored on a 5-point Likert scale. 
Scores range from 17 to 85 with higher scores signifying greater asthma knowledge.

\section{Statistical analysis}

Medians and inter quartile ranges are presented for continuous variables and the number and proportion of participants are presented for categorical variables. Spearman's correlation coefficients are presented to assess the relationship between continuous variables (PSS, child age at asthma diagnosis, BMQ sub scales, PAMSE-13 sub scales, MOSSSS scale and asthma knowledge score) and cockroach KAP. Differences in KAP score by categorical variables, race (black/AA v other), caregiver relationship to child (mother $\mathrm{v}$ other), caregiver education level (greater than high school, high school, or less than high school), home ownership (rent $\mathrm{v}$ own), seen roaches in the previous year (Yes v No), and type of dwelling (detached house, attached house, apartment) were analyzed using the Kruskal-Wallis test. Cockroach KAP scores were categorized into quartiles and associations with natural log transformed cockroach counts were assessed using linear regression. For all analyses, two sided tests were assumed and $p$-values $<0.05$ were considered significant.

\section{Results}

Fifty-six caregivers were recruited into the study (Table 2). The majority identified as Black/African American (88\%) and were mothers of the child with asthma (72\%). Of the participants that agreed to a home visit $(N=32)$, most reported renting their home $(75 \%)$ and almost all reported seeing cockroaches in their home in the previous year (90\%). Participants had positive scores on all subscales of the cockroach KAP survey with knowledge having a relatively lower score than attitudes and practices. For the BMQ the highest score was in the necessity scale (19 out of 25 ) with the harm scale having the lowest relative score (12 out of 20). The correlation between psycho-social and socio-demographic variables and KAP score are presented in Table 3. Cockroach knowledge scores were significantly inversely correlated with age at asthma diagnosis. Cockroach attitude scores were significantly correlated with the BMQ necessity scale, and cockroach practice scores had a significantly moderate correlation with PAMSE-13 attack management sub-scale. The total KAP scale was also significantly correlated with PAMSE-13 attack management scale. Total KAP score was not significantly associated with the concern, overuse, or harm BMQ subscales. The BMQ concern, overuse, and harm scales all had significant inverse correlations with asthma knowledge. For categorical variables (Table 4) caregivers identifying as Black/African American had higher cockroach knowledge scores compared to other races (Median: 6.0 v 3.0; $p=0.05)$ and caregivers other than mothers had higher
Table 2 Characteristics of participants $(N=56)$

\begin{tabular}{|c|c|c|}
\hline & $\mathrm{n}$ & Median (IQR) \\
\hline Age of Child at Asthma Diagnosis & 51 & $3(1-6)$ \\
\hline Roach Number & 26 & $21.5(8-80)$ \\
\hline Perceived Stress Scale & 26 & $7(4-10)$ \\
\hline Medicine Beliefs Questionnaire & 55 & \\
\hline Necessity Scale & & $19(14-22)$ \\
\hline Concerns Scale & & $14(10-19)$ \\
\hline Overuse Scale & & $14(10-16)$ \\
\hline Harm Scale & & $12(10-13)$ \\
\hline Asthma Knowledge & 55 & $54(49-57)$ \\
\hline Cockroach KAP & 56 & \\
\hline Knowledge Scale & & $6(2-8)$ \\
\hline Attitude Scale & & $4.75(2.25-6.00)$ \\
\hline Practice Scale & & $6.5(4.0-8.0)$ \\
\hline Cockroach KAP Total & & $15.25(10-19.25)$ \\
\hline Asthma Self-Efficacy & 26 & \\
\hline Management & & $32(30-34)$ \\
\hline Prevention & & $28(26-30)$ \\
\hline \multirow[t]{2}{*}{ Social Support } & 26 & $28.5(25.0-30.0)$ \\
\hline & $\mathrm{n}$ & n (\%) \\
\hline Race/Ethnicity & 56 & \\
\hline Hispanic all Races & & $3(5.35)$ \\
\hline White & & $5(8.93)$ \\
\hline Black/African American & & $49(87.5)$ \\
\hline Other & & $2(3.57)$ \\
\hline Relationship to Child & 54 & \\
\hline Mother & & $39(72.2)$ \\
\hline Father & & $4(7.4)$ \\
\hline Grandmother & & $10(18.5)$ \\
\hline Aunt & & $1(1.85)$ \\
\hline Education & 56 & \\
\hline Less than High School & & $7(12.5)$ \\
\hline High School/GED & & $21(37.5)$ \\
\hline Greater than High School & & $28(50.0)$ \\
\hline Marital Status & 56 & \\
\hline Married & & $9(16.1)$ \\
\hline Divorced & & $9(16.1)$ \\
\hline Single & & $37(66.1)$ \\
\hline Widowed & & $1(1.79)$ \\
\hline \multicolumn{3}{|l|}{ Home Ownership } \\
\hline Rent Home & 32 & $24(75.0)$ \\
\hline Seen Roaches Past Year & 30 & $27(90.0)$ \\
\hline Type of Dwelling & 31 & \\
\hline Detached House & & $12(38.7)$ \\
\hline Duplex/Triplex & & $11(35.5)$ \\
\hline Row House & & $2(6.5)$ \\
\hline Low Rise Apartment & & $6(19.4)$ \\
\hline
\end{tabular}




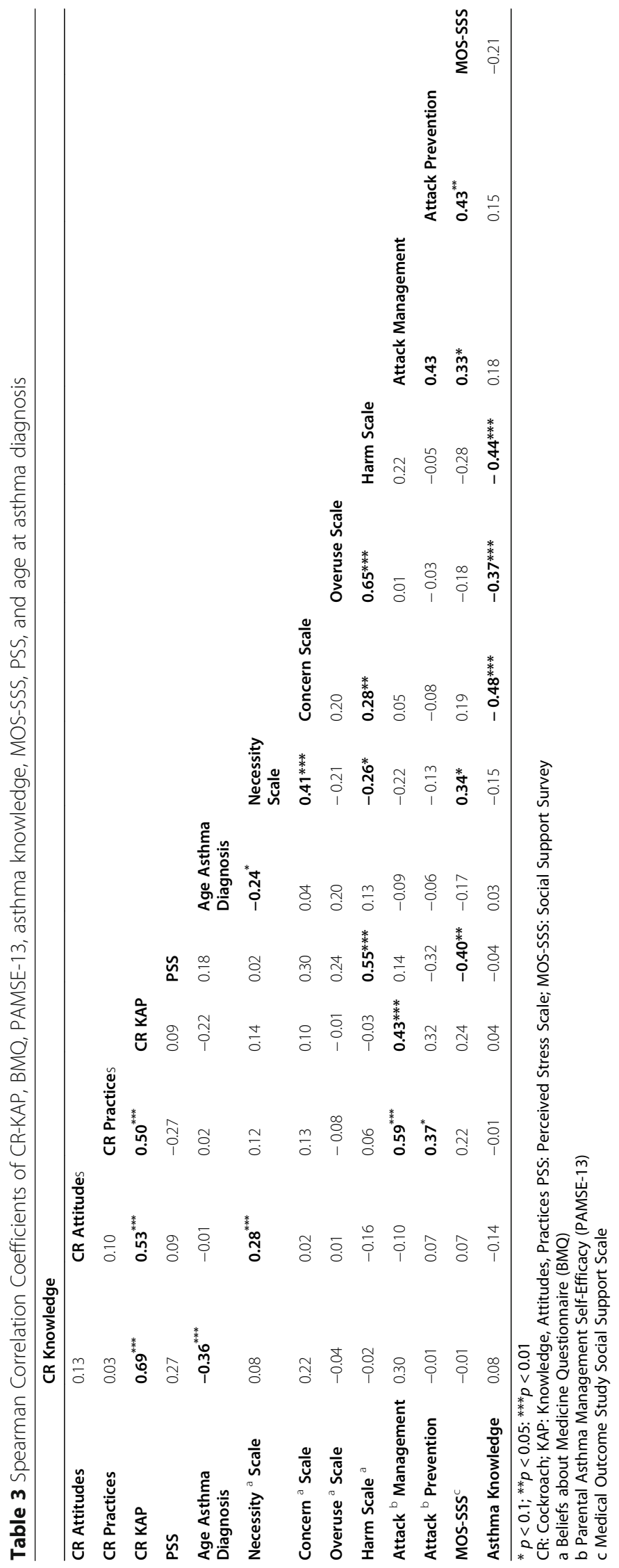


Table 4 Bivariate Association of CR-KAP and Sociodemographic factors

\begin{tabular}{|c|c|c|c|c|c|c|c|c|}
\hline & \multicolumn{2}{|l|}{ CR Knowledge } & \multicolumn{2}{|l|}{ CR Attitudes } & \multicolumn{2}{|l|}{ CR Practices } & \multicolumn{2}{|l|}{ CR KAP Total } \\
\hline & Median (95\% Cl) & $\mathrm{p}$ & Median (95\% Cl) & $p$ & Median $(95 \% \mathrm{Cl})$ & $p$ & Median $(95 \% \mathrm{Cl})$ & $p$ \\
\hline Race/Ethnicity & & 0.05 & & 0.11 & & 0.14 & & 0.84 \\
\hline Black/African American & $6(4-6)$ & & $4(3.5-5)$ & & $6(6-8)$ & & $15(12.5-17)$ & \\
\hline Other & $3(-4-4)$ & & $5.5(4.5-9)$ & & $8(2-10)$ & & $16(9.5-21)$ & \\
\hline Relationship to child & & 0.62 & & 0.003 & & 0.28 & & 0.05 \\
\hline Mother & $6(2-6)$ & & $4(3-5)$ & & $6(5-8)$ & & $14(11-16)$ & \\
\hline Other & $4(4-8)$ & & $6.5(5-8)$ & & $8(6-8)$ & & $18(15-20.5)$ & \\
\hline Education & & 0.81 & & 0.16 & & 0.09 & & 0.54 \\
\hline Less than $\mathrm{HS}$ & $4(-4-8)$ & & $4(2-6)$ & & $8(6-10)$ & & $16(11-21.5)$ & \\
\hline HS & $4(2-6)$ & & $3(2-6)$ & & $6(4-8)$ & & $14.5(9.5-16.5)$ & \\
\hline Greater than HS & $6(4-6)$ & & $5(4-6)$ & & $6(4-8)$ & & 16.5 (10-19.5) & \\
\hline Partnered & & 0.22 & & 0.52 & & 0.32 & & 0.5 \\
\hline Yes & $8(0-8)$ & & $5(1.5-7.5)$ & & $6(4-8)$ & & $17(9.5-20.5)$ & \\
\hline No & $4(2-6)$ & & $4.5(3.5-5)$ & & $7(6-8)$ & & $15(12-16.5)$ & \\
\hline Home Ownership & & 0.64 & & 0.69 & & 0.64 & & 0.79 \\
\hline Own Home & $5(2-10)$ & & $4.25(2-8.5)$ & & $7(3-10)$ & & $15.25(11-26.5)$ & \\
\hline Rent Home & $6(2-6)$ & & $4(3-5)$ & & $7.5(6-8)$ & & $15.5(12-18)$ & \\
\hline Seen Roaches Past Year & & 0.81 & & 0.43 & & 0.73 & & 0.78 \\
\hline Yes & $6(2-6)$ & & $4(3-5)$ & & $7(6-8)$ & & $15(12-18)$ & \\
\hline No & $6(2-8)$ & & $3.5(3-3.5)$ & & $7(4-10)$ & & $13.5(12-21.5)$ & \\
\hline Type of Dwelling & & 0.83 & & 0.79 & & 0.19 & & 0.19 \\
\hline Detached House & $4(2-8)$ & & $4.25(2-6.5)$ & & $7(4-8)$ & & $16(10-20.5)$ & \\
\hline Attached House & $6(-4-8)$ & & $4(2-5)$ & & $6(3-8)$ & & $15(7-17)$ & \\
\hline Apartment & $6(2-8)$ & & $4.25(1.5-8)$ & & $8(3-10)$ & & $18.25(12-22)$ & \\
\hline
\end{tabular}

cockroach attitudes (Median 6.5 v $4.0 ; p=0.003$ ) and total KAP scores (Median $18.0 \mathrm{v} 14.0 ; p=0.05$ ). Twentysix participants completed the home visit (Table 5). The number of cockroaches in the home was not associated with greater cockroach knowledge, cockroach practice or total KAP score. The highest quartile consistently had fewer cockroaches compared to the lowest quartile although results did not reach statistical significance. For cockroach attitude scores, participants in the highest quartile had significantly fewer cockroaches trapped ( $\beta$ : $-1.96,95 \%$ CI: $-3.50,-0.42)$ compared to those with scores in the lowest quartile $(p=0.01)$.

\section{Discussion}

Reducing cockroach exposure in the home is recommended as an asthma management strategy [12] Adherence to this recommendation by caregivers of children with asthma is low and the reason for low adherence is unclear. The current study was conducted to address this data gap. We found that greater knowledge about cockroaches was associated with lower age at asthma diagnosis indicating that caregivers had less knowledge about the importance of cockroach exposure the older the child was when diagnosed. This is an important finding suggesting the need for greater emphasis on cockroach control in the home of older children with asthma. Greater concern about cockroach exposure (attitudes) was significantly correlated with the BMQ necessity scale. These results indicate that caregivers who are more concerned about the effect of cockroach exposure on their child's asthma also have positive beliefs about the need for medication adherence. Therefore, caregivers with positive medication beliefs may be more receptive to cockroach interventions to control their child's asthma. Cockroach practice score (reflecting taking action to promote remediation) was associated with both the attack management and attack prevention sub-scales of the PAMSE-13. These are promising results. Caregivers who indicated they had greater self-efficacy to manage and prevent asthma attacks reported practicing the actions required to reduce cockroach exposure. Taken together, the results indicate that the number of cockroaches in the home is most closely associated with how concerned (attitudes) caregivers are about 
Table 5 Association between natural log roach number and CR-KAP Scales

\begin{tabular}{clc}
\hline & $\boldsymbol{\beta} \mathbf{( 9 5 \% \mathrm { Cl } )}$ & $\mathbf{p}$ \\
\hline Cockroach Knowledge & & 0.82 \\
Q1 & Ref & \\
Q2 & $0.10(-2.67,2.86)$ & \\
Q3 & $0.59(-2.12,3.3)$ & \\
Q4 & $-0.26(-3.12,2.59)$ & $\mathbf{0 . 0 1}$ \\
Cockroach Attitudes & & \\
Q1 & Ref & \\
Q2 & $-1.69(-3.49,0.12)$ & \\
Q3 & $0.49(-1.00,1.98)$ & \\
Q4 & $-1.96(-3.50,-0.42)$ & \\
Cockroach Practices & & \\
Q1 & Ref & \\
Q2 & $-1.73(-3.99,0.54)$ & \\
Q3 & $-1.48(-3.40,0.43)$ & \\
Q4 & $-1.65(-4.23,0.93)$ & \\
CR-KAP Total & & \\
Q1 & Ref & \\
Q2 & $-0.30(-2.72,2.12)$ & \\
Q3 & $0.23(-1.83,2.29)$ & \\
Q4 & $-0.92(-2.98,1.14)$ & \\
\hline
\end{tabular}

cockroaches and their control as opposed to what they know (knowledge) or what they reported doing (practices) to prevent cockroaches.

These results reveal that higher practice scores did not significantly reduce cockroach exposure although participants in the higher quartiles had fewer cockroaches compared to those in the lowest quartile. This could be due to social desirability bias. Participants may have overestimated their responses on the practice scale due to perceived stigma around having pests in the home. Any bias would likely be non-differential since cockroach KAP was assessed prior to cockroach trapping and almost all participants reported seeing roaches in the home. Another reason may be that attitudes are a better indicator of health behavior than reported practices. This finding would align with previous research evaluating the HBM where perceived barriers and benefits (i.e. attitudes) are the strongest predictor of behavior change.

Another interesting finding is that the cockroach KAP subscales were not significantly correlated with asthma knowledge. This contrasts with the BMQ subscales (concern, overuse, and harm) which were moderately correlated with asthma knowledge, suggesting that improving asthma knowledge affects beliefs concerning asthma medications. However, the same is not true for cockroach control which is often overlooked as a strategy for managing asthma. Improving knowledge (either asthma or cockroach) may not be sufficient to increase concern (attitudes) or improve cockroach remediation practices. Finally, although participants in the highest quartile of total KAP had fewer cockroaches compared to those in the lowest quartile, the overall KAP score was not associated with reduced cockroach exposure. This suggests that the individual components may be more important than their sum.

Overall, the results provide information on key factors as targets for improving asthma management among caregivers with the intention of improving cockroach avoidance strategies. There appears to be patterns of different groups of caregivers which may require different avenues of support.

The study had several strengths. Participants were recruited from both community events and pulmonary clinics which allowed enrollment of participants specifically seeking care as well as those not seeking care. The cohort study was a prospective design and cockroach exposure was assessed after the assessment of cockroach KAP reducing the likelihood of information bias. In addition, actual cockroach exposure was assessed using cockroach counts. Limitations include lack of data on all sociodemographic factors that may influence both the home environment and cockroach exposure including household income, the small sample size which resulted in the inability to control for potential confounding factors, especially when assessing cockroach exposure, therefore the findings may be the result of residual confounding and should be considered with caution. Small sample size also resulted in reduced power. Finally, the use of convenience sampling may result in reduced generalizability.

\section{Conclusion}

Generally, caregivers of children had positive scores on the cockroach KAP survey indicating positive heath behavior knowledge, attitudes, and practices. The hypothesis that greater knowledge, attitude, and practice scores would be associated with reduced cockroach exposure in the home was not entirely supported. Greater cockroach knowledge and practice scores were not associated with reduced cockroach exposure. Cockroach attitude scores appear to be driving exposure to cockroach in homes of children with asthma.

\section{Abbreviations}

U.S.: United States; HBM: Health Beliefs Model; KAP: Knowledge, Attitudes, and Practices; BMQ: Beliefs about Medicine Questionnaire; PSS: Perceived Stress Scale; PAMSE-13: Parental Asthma Management Self-Efficacy; MOSSSS: Medical Outcome Study Social Support Scale

\section{Acknowledgements}

We would like to thank Children's Hospital of New Orleans (CHNOLA) and our community partners for assisting with recruitment. In addition, we would like to thank the participants for their involvement in the study. 


\section{Authors' contributions}

DW contribution was to the conception, design, interpretation, and analysis of the data and was a major contributor in writing the manuscript. FR was substantial contribution to the conception, acquisition, interpretation of the data, and was a major contributor in writing the manuscript. CR contributed to the acquisition of data and was a contributor in writing the manuscript. All authors have read and approved the final manuscript and agree to be accountable for their contribution to the manuscript.

\section{Funding}

The study was funded by grant number $555599 \mathrm{G} 1$ by the Charles and Elizabeth Wetmore Fund.

\section{Availability of data and materials}

The datasets used and/or analyzed during the current study are available from the corresponding author on reasonable request.

\section{Declarations}

\section{Ethics approval and consent to participate}

The study was approved by Tulane University's Biomedical Institutional Review Board. All participants were consented before data collection. Informed consent was obtained from all subjects prior to data collection. All methods were carried out in accordance with relevant guidelines and regulations.

\section{Consent for publication}

Not applicable.

\section{Competing interests}

The authors declare that they have no competing interests.

Received: 9 March 2021 Accepted: 14 June 2021

Published online: 29 July 2021

\section{References}

1. Ferrante G, La Grutta S. The burden of pediatric asthma. Front Pediatr. 2018; 6:186. https://doi.org/10.3389/fped.2018.00186

2. Gray WN, Netz M, McConville A, Fedele D, Wagoner ST, Schaefer MR. Medication adherence in pediatric asthma: a systematic review of the literature. Pediatr Pulmonol. 2018;53(5):668-84. https://doi.org/10.1002/ ppul.23966.

3. Nurmagambetov T, Kuwahara R, Garbe P. The economic burden of asthma in the United States, 2008-2013. Ann Am Thoracic Soc. 2018;15(3):348-56. https://doi.org/10.1513/AnnalsATS.201703-2590C.

4. Perry R, Braileanu G, Palmer T, Stevens P. The economic burden of pediatric asthma in the United States: literature review of current evidence. Pharmacoeconomics. 2019:37(2):155-67. https://doi.org/10.1007/s40273-0180726-2.

5. Le Cann P, Paulus H, Glorennec P, Le Bot B, Frain S, Gangneux JP. Home environmental interventions for the prevention or control of allergic and respiratory diseases: what really works. J Allergy Clin Immunol Pract. 2017; 5(1):66-79. https://doi.org/10.1016/j.jaip.2016.07.011.

6. Pomés A, Mueller GA, Randall TA, Chapman MD, Arruda LK. New insights into cockroach allergens. Curr Allergy Asthma Rep. 2017;17(4):25. https://doi. org/10.1007/s11882-017-0694-1.

7. Rabito FA, Carlson J, Holt EW, labal S, James MA. Cockroach exposure independent of sensitization status and association with hospitalizations for asthma in inner-city children. Ann Allergy Asthma Immunol. 2011;106(2): 103-9. https://doi.org/10.1016/j.anai.2010.10.013.

8. Togias A, Fenton MJ, Gergen PJ, Rotrosen D, Fauci AS. Asthma in the inner city: the perspective of the National Institute of Allergy and Infectious Diseases. J Allergy Clin Immunol. 2010;125(3):540-4. https://doi.org/10.1016/ j.jaci.2010.01.040

9. Rabito FA, Iqbal S, Holt E, Grimsley LF, Islam TM, Scott SK. Prevalence of indoor allergen exposures among New Orleans children with asthma. J Urban Health. 2007;84(6):782-92. https://doi.org/10.1007/s11524-007-9216-0.

10. Rosenstreich DL, Eggleston P, Kattan M, Baker D, Slavin RG, Gergen P, et al. The role of cockroach allergy and exposure to cockroach allergen in causing morbidity among inner-city children with asthma. N Engl J Med. 1997;336(19):1356-63. https://doi.org/10.1056/NEJM199705083361904.
11. Do DC, Zhao Y, Gao P. Cockroach allergen exposure and risk of asthma. Allergy. 2016;71(4):463-74.

12. Expert Panel Report 3 (EPR-3). Guidelines for the Diagnosis and Management of Asthma-Summary Report 2007. J Allergy Clin Immunol. 2007;120(5):S94-S138.

13. Matsui EC. Environmental exposures and asthma morbidity in children living in urban neighborhoods. Allergy. 2014;69(5):553-8. https://doi.org/10.1111/a II.12361.

14. Zahran HS, Bailey CM, Damon SA, Garbe PL, Breysse PN. Vital signs: asthma in children-United States, 2001-2016. Morb Mortal Wkly Rep. 2018;67(5): 149-55. https://doi.org/10.15585/mmwr.mm6705e1.

15. Kader R, Kennedy K, Portnoy JM. Indoor environmental interventions and their effect on asthma outcomes. Curr Allergy Asthma Rep. 2018;18(3):17. https://doi.org/10.1007/s11882-018-0774-x.

16. McQuaid EL, Landier W. Cultural issues in medication adherence: disparities and directions. J Gen Intern Med. 2018;33(2):200-6. https://doi.org/10.1007/ s11606-017-4199-3.

17. Felicia AR, John CC, Hua H, Derek W, Coby S. A single intervention for cockroach control reduces cockroach exposure and asthma morbidity in children. J Allergy Clin Immunol. 2017;140:565-70.

18. Gumucio S, Luhmann N, Fauvel G, Zompi S, Ronsse A, Courcaud A, et al. The KAP survey model: knowledge, attitude, and practices. Saint-Etienne: IGC Communigraphie; 2011. p. 4-5.

19. Rosenstock I. Why people use health services. The Milbank Memorial Fund Quarterly. 1966;44(3):94-127. https://doi.org/10.2307/3348967.

20. Carpenter CJ. A meta-analysis of the effectiveness of health belief model variables in predicting behavior. Health Commun. 2010;25(8):661-9. https:// doi.org/10.1080/10410236.2010.521906.

21. Harrison JA, Mullen PD, Green LW. A meta-analysis of studies of the health belief model with adults. Health Educ Res. 1992;7(1):107-16. https://doi. org/10.1093/her/7.1.107.

22. Rosenstock IM, Strecher VJ, Becker MH. Social learning theory and the health belief model. Health Educ Q. 1988;15(2):175-83. https://doi.org/10.11 77/109019818801500203.

23. Campbell ME, Dwyer JJ, Goettler F, Ruf F, Vittiglio M. A program to reduce pesticide spraying in the indoor environment: evaluation of the 'roach Coach'project. Can J Public Health. 1999;90(4):277-81. https://doi.org/10.1 007/BF03404131.

24. Arbes SJ, Sever M, Mehta J, Gore JC, Schal C, Vaughn B, et al. Abatement of cockroach allergens (Bla g 1 and Bla g 2) in low-income, urban housing ismonth 12 continuation results. J Allergy Clin Immunol. 2004; 113(1):109-14. https://doi.org/10.1016/j.jaci.2003.10.042.

25. Cohen S, Kamarck T, Mermelstein R. A global measure of perceived stress. J Health Soc Behav. 1983;24(4):385. https://doi.org/10.2307/2136404.

26. Holden L, Lee C, Hockey R, Ware RS, Dobson AJ. Validation of the MOS social support survey 6-item (MOS-SSS-6) measure with two large population-based samples of Australian women. Qual Life Res. 2014;23(10): 2849-53. https://doi.org/10.1007/s11136-014-0741-5.

27. Horne R, Weinman J. Patients' beliefs about prescribed medicines and their role in adherence to treatment in chronic physical illness. J Psychosom Res. 1999;47(6):555-67. https://doi.org/10.1016/S0022-3999(99)00057-4.

28. Bursch B, Schwankovsky L, Gilbert J, Zeiger R. Construction and validation of four childhood asthma self-management scales: parent barriers, child and parent self-efficacy, and parent belief in treatment efficacy. J Asthma. 1999; 36(1):115-28. https://doi.org/10.3109/02770909909065155.

29. Landeo-Gutierrez J, Celedón JC. Chronic stress and asthma in adolescents. Ann Allergy Asthma Immunol. 2020;125(4):393-8.

30. Martínez CR, Sossa M. Validation of an asthma knowledge questionnaire for use with parents or guardians of children with asthma. Archivos de Bronconeumología ((English Edition)). 2005;41(8):419-24.

\section{Publisher's Note}

Springer Nature remains neutral with regard to jurisdictional claims in published maps and institutional affiliations. 